\title{
Foraging habits of reef fishes associated with mangroves and seagrass beds in a Caribbean lagoon: A stable isotope approach
}

\section{Hábitos de alimentación de peces arrecifales asociados con manglares y pastos marinos en una laguna del Caribe: Un enfoque de isótopos estables}

\author{
Amandine Vaslet ${ }^{1 *}$, Yolande Bouchon-Navaro ${ }^{1}$, Mireille Harmelin-Vivien ${ }^{2}$, Gilles Lepoint ${ }^{3}$, \\ Max Louis ${ }^{1}$, Claude Bouchon ${ }^{1}$ \\ ${ }^{1}$ UMR BOREA-DYNECAR, Université Antilles-Guyane Campus de Fouillole, BP592, 97159 Pointe-à-Pitre, \\ Guadeloupe, France. \\ 2 Institut Méditerranéen d'Océanologie (MIO), CNRS/INSU, Université Aix-Marseille, Campus de Luminy, \\ 13288, Marseille Cedex 9, France. \\ ${ }^{3}$ MARE Centre, Laboratoire d'Océanologie, Université de Liège, Sart Tilman, 4000 Liège, Belgium. \\ * Corresponding author. E-mail: amandine.vaslet@univ-ag.fr
}

\begin{abstract}
Mangroves and seagrass beds represent suitable fish habitats as nurseries or feeding areas. This study was conducted in a Caribbean lagoon to assess the foraging habits of juvenile transient reef fishes in these two habitats. Twelve fish species were sampled in coastal mangroves, an offshore mangrove islet, and a seagrass bed site, and stable isotope analyses were performed on fishes and their prey items. The SIAR mixing model indicated that transient fishes from both mangroves and seagrass beds derived most of their food from seagrass beds and their associated epiphytic community. Only a few species including planktivores (Harengula clupeola, Anchoa lyolepis) and carnivores (Centropomus undecimalis and small specimens of Ocyurus chrysurus) presented depleted carbon values, showing reliance on mangrove prey in their diets. Mangrove-derived organic matter contributed marginally to the diet of transient fishes, which relied more on seagrass food sources. Thus, mangroves seem to function more as refuge than feeding habitats for juvenile transient fishes.
\end{abstract}

Keys words: mangrove, seagrass, fish, stable isotopes, SIAR.

RESUMEN. Los manglares y las praderas de pastos marinos son hábitats adecuados para la reproducción y alimentación de peces. En este estudio se evaluaron los hábitos alimentarios de peces arrecifales juveniles que transitan por estos dos tipos de hábitat en una laguna del Caribe. Se recolectaron 12 especies de peces en los manglares costeros, un islote de manglares y un sitio de praderas de pastos marinos, y se realizaron análisis de isótopos estables en los peces y sus presas. El modelo SIAR indicó que los peces transeúntes se alimentaron principalmente de los pastos marinos y su comunidad epifita asociada. Sólo unas cuantas especies incluyendo peces planctívoros (Harengula clupeola, Anchoa lyolepis) y carnívoros (Centropomus undecimalis y pequeños ejemplares de Ocyurus chrysurus) presentaron valores de carbono empobrecidos, lo que indica una dependencia de las presas de manglares para sus dietas. La materia orgánica producida en el manglar contribuyó poco a la dieta de los peces transeúntes, los cuales dependieron más de fuentes provenientes de los pastos marinos. Por lo tanto, los peces juveniles transeúntes usan los manglares más como refugio que como hábitat de alimentación.

Palabras clave: manglares, pastos marinos, peces, isótopos estables, modelo SIAR.

\section{INTRODUCTION}

Mangroves and seagrass beds constitute highly productive ecosystems that are known to represent suitable fish habitats, acting as shelters, nurseries or foraging areas (Beck et al. 2001, Nagelkerken et al. 2008). The protection against predation, the interception of planktonic fish larvae, and the abundance of food sources are among the main assumptions explaining the high abundance of juvenile reef fishes in these habitats (Beck et al. 2001, Laegdsgaard and Johnson 2001). In the Caribbean, mangroves are often interlinked with seagrass beds and the low tidal influence enables permanent exchanges between these habitats through diurnal or nocturnal fish migrations (Nagelkerken et al. 2000, Kopp et al.

\section{INTRODUCCIÓN}

Los manglares y las praderas de pastos marinos son ecosistemas altamente productivos así como hábitats adecuados para el refugio, la crianza y la alimentación de peces (Beck et al. 2001, Nagelkerken et al. 2008). La protección contra depredadores, la interceptación de larvas planctónicas de peces y la abundancia de fuentes de alimento son algunas de las principales hipótesis que explican la gran abundancia de individuos juveniles de peces arrecifales en estos hábitats (Beck et al. 2001, Laegdsgaard y Johnson 2001). En el Caribe, los manglares frencuentemente se interrelacion con las praderas de pastos marinos y la poca influencia de las mareas permite un intercambio permanente entre estos 
2007). Mangrove ecosystems can be classified into several categories including fringing mangroves that occur along sheltered coastlines or mangrove islets (Gilmore and Snedaker 1993, Ewel et al. 1998). The configuration and structure of these fringing mangroves can vary substantially among locations and have a direct influence on the function and role of these coastal habitats (Ewel et al. 1998, Heithaus et al. 2011).

Feeding activities of transient fishes, comprising species that are present in mangroves during their juvenile life stages, can represent linkages between mangroves and adjacent coastal habitats (Heithaus et al. 2011). Many fish species are characterized as opportunistic species because their diets may change according to the availability and abundance of prey items (Laegdsgaard and Johnson 2001).

Stable isotopes have been used as proxies for contributions of mangrove and seagrass prey to fish diets and have shown marginal contribution of mangrove-derived organic matter (Nagelkerken et al. 2008). Transient fishes in mangroves primarily feed on allochthonous organic matter derived from plankton, algae, or seagrass beds (Marguillier et al. 1997, Kieckbusch et al. 2004, Nyunja et al. 2009). Yet, mangrove and seagrass food source contributions to fish diets seem to be site-specific and depend on hydrological processes, including tides and currents, or habitat structure (Lugendo et al. 2007, Granek et al. 2009, Vaslet et al. 2012). In Tanzania, Lugendo et al. (2007) found that fishes remaining in enclosed mangrove ponds during low tides derived most of their food from this habitat, whereas fishes from open fringing mangroves migrated and fed in adjacent inundated seagrass beds. Tidal range is therefore an important factor in the Indo-Pacific area because it drives trophic migration of fish species from subtidal habitats to inundated mangroves (Sheaves and Molony 2000, Lugendo et al. 2007). However, this environmental descriptor is less relevant in the Lesser Antilles region, where tides are generally lower than $1 \mathrm{~m}$ and mangroves are continuously flooded (Nagelkerken et al. 2008). Therefore, spatial structure (in terms of location and landscape characteristics of mangrove sites) may be of primary importance while assessing the origin of carbon sources assimilated by fishes (Vaslet et al. 2012).

This study considered different fringing mangroves (i.e., offshore mangrove islet, coastal mangroves) in a Caribbean lagoon and investigated the primary food sources of transient juvenile fishes. We examined the influence of mangrove location and habitat structure on the feeding behavior of 12 juvenile transient fishes by analyzing spatial patterns of stable isotopes. Carbon and nitrogen isotopic compositions provide good insights into the main food sources assimilated by fishes the preceding weeks (Gearing 1991). As a result of different inorganic carbon sources and photosynthesis, carbon isotopic fingerprints of mangroves are on average more depleted in ${ }^{13} \mathrm{C}$ than are those of seagrass beds (Hemminga and Mateo 1996, Nagelkerken and van der Velde 2004a). habitats mediante las migraciones diurnas y nocturnas de peces (Nagelkerken et al. 2000, Kopp et al. 2007). Existen diferentes tipos de manglares entre ellos los manglares de borde que se desarrollan a lo largo de los márgenes de costas protegidas o islotes de manglares (Gilmore y Snedaker 1993, Ewel et al. 1998). La configuración y estructura de estos manglares de borde puede variar considerablemente entre localidades y tener una influencia directa en la función y papel de estos hábitats costeros (Ewel et al. 1998, Heithaus et al. 2011).

Las actividades de alimentación de peces transeúntes, esto es, especies que se encuentran en manglares durante su etapa juvenil, pueden representar un vínculo entre los manglares y los hábitats costeros adyacentes (Heithaus et al. 2011). Varias especies de peces se caracterizan como especies oportunistas ya que su dieta puede cambiar según la disponibilidad y abundancia de presas (Laegdsgaard y Johnson 2001).

Los isótopos estables han sido usados como marcadores de las contribuciones de las presas procedentes de manglares y pastos marinos a las dietas de peces y han mostrado una contribución marginal de la materia orgánica proveniente de manglares (Nagelkerken et al. 2008). Los peces que transitan por los manglares se alimentan principalmente de materia orgánica alóctona derivada de plancton, algas o pastos marinos (Marguillier et al. 1997, Kieckbusch et al. 2004, Nyunja et al. 2009). No obstante, las contribuciones de las fuentes de alimento de manglares y pastos marinos parece depender del sitio así como de la estructura del hábitat o de procesos hidrológicos, incluyendo las mareas y corrientes (Lugendo et al. 2007, Granek et al. 2009, Vaslet et al. 2012). En Tanzania, Lugendo et al. (2007) encontraron que los peces que permanecían en manglares encerrados durante la marea baja obtenían la mayor parte de su alimento en este hábitat, mientras que los peces de manglares de bordo abiertos migraban y se alimentaban en las praderas de pastos marinos inundados. Por lo tanto, el intervalo de marea es un factor importante en la región indo-pacífica ya que influye en la migración trófica de especies de peces de hábitats submareales a manglares inundados (Sheaves y Molony 2000, Lugendo et al. 2007). En las Antillas Menores, sin embargo, donde las mareas generalmente son $<1 \mathrm{~m}$ y los manglares se encuentran continuamente inundados, este descriptor ambiental es menos relevante (Nagelkerken et al. 2008), y la estructura espacial (en términos de ubicación y las características fisiográficas de los manglares) podría resultar de gran importancia en la evaluación del origen de las fuentes de carbono que asimilan los peces (Vaslet et al. 2012).

En este trabajo se estudiaron diferentes manglares de borde (i.e., un islote de manglares y manglares costeros) en una laguna del Caribe y se analizaron las principales fuentes de alimento de los peces juveniles transeúntes. Se examinó la influencia de la ubicación de los manglares y la estructura del hábitat en el comportamiento alimentario de 12 peces juveniles transeúntes mediante los patrones espaciales de isótopos 
Carbon isotopes can thus be used as proxies to investigate the habitat from which fish species derive their diet. Trophic levels of consumers in food webs are revealed by nitrogen values (Peterson and Fry 1987). Consequently, stable isotope analyses enable tracing mangrove- and seagrass-derived organic matter in coastal food webs and assessment of trophic linkages between these habitats.

The objectives of the present study were (1) to investigate the influence of habitat structure on the feeding behavior of juvenile transient fishes and (2) to examine potential variations in fish feeding behavior regarding their feeding strategy (i.e., trophic category) and life stage (i.e., juveniles, adults).

\section{MATERIALS AND METHODS}

\section{Study location}

The study was carried out in Grand Cul-de-Sac Marin (GCSM), a bay on Guadeloupe Island (Lesser Antilles) (fig. 1). A barrier reef encloses this bay and delimits a lagoon of 11,000 ha. The shoreline is fringed by Rhizophora mangle (Linnaeus 1753) mangroves with prop-roots always inundated. The GCSM lagoon presents large areas of seagrass beds, mainly composed of Thalassia testudinum Banks and Soland ex König, 1805 and Syringodium filiforme (Kütz 1860), covering about 8200 ha (Chauvaud et al. 2001). With the low tidal amplitude ( 0.5 to $0.6 \mathrm{~m}$ ) occurring in the area (Assor 1988), mangroves are permanently flooded. Water depth varies from a few decimeters on shallows and coral keys to $30 \mathrm{~m}$ in reef passes. Salinity in the GCSM lagoon varies between 20.1 near river mouths to 36.5 around mangrove islets close to the barrier reef (Fajou and Carenage offshore islets; Vaslet et al. 2010). Three sites were studied between September and December 2007: Fajou offshore mangrove islet located near the barrier reef (site F), coastal fringing mangroves located close to the mouth of Grande Rivière à Goyaves (site $\mathrm{G}$ ), and a seagrass bed in the middle of the GCSM lagoon (site S) (fig. 1). The mangrove forest is more developed at site $\mathrm{G}$ than at site $\mathrm{F}$, with a forest width of $1.2 \mathrm{~km}$ and $760 \mathrm{~m}$, respectively (Vaslet et al. 2010).

\section{Study design}

Fishes were collected from mangroves using fish traps (see description in Vaslet et al. 2010) and from seagrass beds with a seine net, composed of a bag ( $3 \mathrm{~m}$ long, $2 \mathrm{~m}$ high) with two wings ( $23 \mathrm{~m}$ long, $2 \mathrm{~m}$ high) (Bouchon-Navaro et al. 1992). Twelve transient fish species of similar size ranges were collected at the three sites. The fish species Bairdiella ronchus (Cuvier 1830) was also sampled to examine food source contributions to the diet of this resident species that spends its entire life cycle in mangroves (Louis 1985).

Fish species were assigned to four trophic guilds based on dietary analyses of fish gut contents (table 1) and a review of estables. La composición isotópica del carbono y el nitrógeno proporciona información de las principales fuentes del alimento consumido por los peces en las semanas anteriores (Gearing 1991). Como resultado de diferentes fuentes de carbono inorgánico y la fotosíntesis, las huellas isotópicas del carbono de los manglares en comparación con los pastos marinos se hallan en promedio más empobrecidos en ${ }^{13} \mathrm{C}$ (Hemminga y Mateo 1996, Nagelkerken y van der Velde 2004a). Por lo tanto, los isótopos del carbono pueden usarse como marcadores para estudiar el hábitat en donde las especies de peces se alimentan. Los valores de nitrógeno muestran los niveles tróficos de los consumidores en las cadenas alimentarias (Peterson y Fry 1987). Por consiguiente, el análisis de isótopos estables permite rastrear la materia orgánica procedente de manglares y pastos marinos en las cadenas alimentarias en zonas costeras y evaluar los vínculos tróficos entre estos hábitats.

Los objetivos del presente estudio fueron (1) investigar la influencia de la estructura del hábitat en el comportamiento alimentario de peces juveniles transeúntes y (2) analizar las variaciones potenciales en el comportamiento alimentario de peces en cuanto a su estrategia de alimentación (i.e., categoría trófica) y etapa de vida (i.e., juveniles, adultos).

\section{MATERIALES Y MÉTODOS}

\section{Área de estudio}

El estudio se realizó en una bahía, Grand Cul-de-Sac Marin (GCSM), de la isla de Guadalupe (Antillas Menores) (fig. 1). Un arrecife encierra esta bahía y delimita una laguna de 11,000 ha. La costa está bordeada por el mangle rojo, Rhizophora mangle (Linnaeus 1753), cuyas raíces siempre se encuentran inundadas. La laguna de GCSM presenta áreas grandes de praderas de pastos marinos, compuestas principalmente por Thalassia testudinum Banks y Soland ex König, 1805 y Syringodium filiforme (Kütz 1860), que cubren alrededor de 8200 ha (Chauvaud et al. 2001). Debido a la baja amplitud mareal (0.5 a $0.6 \mathrm{~m}$ ) en la zona (Assor 1988), los manglares siempre están inundados. La profundidad del agua varía desde unos cuantos decímetros en los bajos y cayos coralinos hasta $30 \mathrm{~m}$ en los canales arrecifales. La salinidad en la laguna de GCSM varía entre 20.1 cerca de las desembocaduras de ríos y 36.5 alrededor de los islotes de manglares (islotes Fajour y Carenage) cerca de la barrera de coral (Vaslet et al. 2010). Se estudiaron tres sitios entre septiembre y diciembre de 2007: el islote de Fajou cerca de la barrera arrecifal (sitio F), manglares de borde cerca de la boca de Grande Rivière à Goyaves (sitio G), y un sitio de praderas de pastos marinos en medio de la laguna de GCSM (sitio S) (fig. 1). El bosque de mangles del sitio G (1.2 km de ancho) es más grande que el del sitio $\mathrm{F}$ (760 $\mathrm{m}$ de ancho) (Vaslet et al. 2010). 


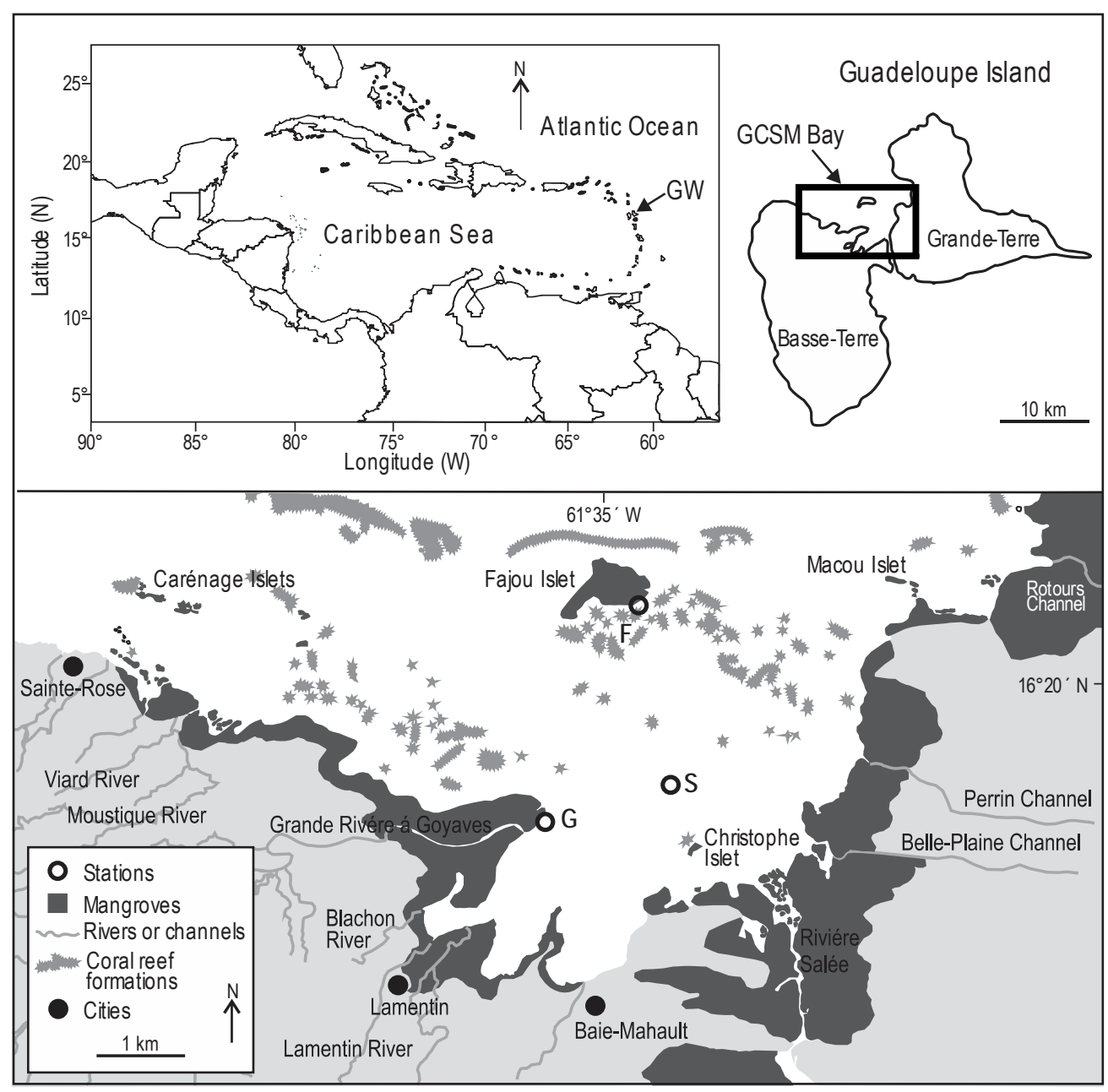

Figure 1. Location of Guadeloupe (GW) in the Caribbean. Location of the three study sites in Grand Cul-de-Sac Marin (GCSM): offshore mangrove islet of Fajou $(\mathrm{F})$, coastal mangroves near Grande Rivière à Goyaves river mouth $(\mathrm{G})$, and a seagrass bed site $(\mathrm{S})$.

Figura 1. Ubicación de la isla de Guadalupe (GW) en el Caribe. Ubicación de los tres sitios de estudio en la bahía llamada Grand Cul-de-Sac Marin (GCSM): islote de Fajou (F), manglares costeros cerca de la desembocadura de Grande Rivière à Goyaves (G) y un sitio de praderas de pastos marinos $(\mathrm{S})$.

literature on fish diets (Randall 1967, Austin and Austin 1971, Aguirre-León and Yáñez-Arancibia 1984, BouchonNavaro et al. 1992, Sierra et al. 1994, Nagelkerken et al. 2006, Chiaverini 2008): herbivores consuming algae, seagrass leaves, and their epiphytic algae; omnivores feeding on invertebrates and at least $10 \%$ of algae; planktivores consuming zooplankton; and carnivores ingesting benthic invertebrates and some fish species.

The following fish prey were collected in mangroves and seagrass beds: mangrove litter, seagrass leaves, epiphytes scraped from seagrass leaves, algae, annelids, gastropods, amphipods, crabs, and shrimps. Samples of seston were collected above the seagrass site and between 10 and $20 \mathrm{~m}$ from mangrove fringes by towing a plankton net $(100 \mu \mathrm{m}$ mesh size) at $0.5 \mathrm{~m}$ below the water surface during $5 \mathrm{~min}$. Zooplankton (dominated by copepods) was sorted from dead suspended materials at the laboratory.

\section{Diseño del estudio}

En los manglares los peces se recolectaron con trampas (ver descripción en Vaslet et al. 2010) y en las praderas de pastos marinos se recolectaron con una red de cerco, que consistió de una bolsa ( $3 \mathrm{~m}$ de largo, $2 \mathrm{~m}$ de alto) con dos alas (23 $\mathrm{m}$ de largo, $2 \mathrm{~m}$ de alto) (Bouchon-Navaro et al. 1992). Se recolectaron 12 especies de peces transeúntes de tamaño similar en los tres sitios. También se capturó el pez Bairdiella ronchus para analizar las fuentes de alimento que forman la dieta de esta especie residente que permanece todo su ciclo de vida en los manglares (Louis 1985).

Las especies de peces se asignaron a cuatro gremios tróficos con base en el análisis de su contenido estomacal (tabla 1) y una revisión de la literatura de dietas de peces (Randall 1967, Austin y Austin 1971, Aguirre-León y YáñezArancibia 1984, Bouchon-Navaro et al. 1992, Sierra et al. 
Vaslet et al.: Foraging habits of juvenile fishes in a Caribbean lagoon

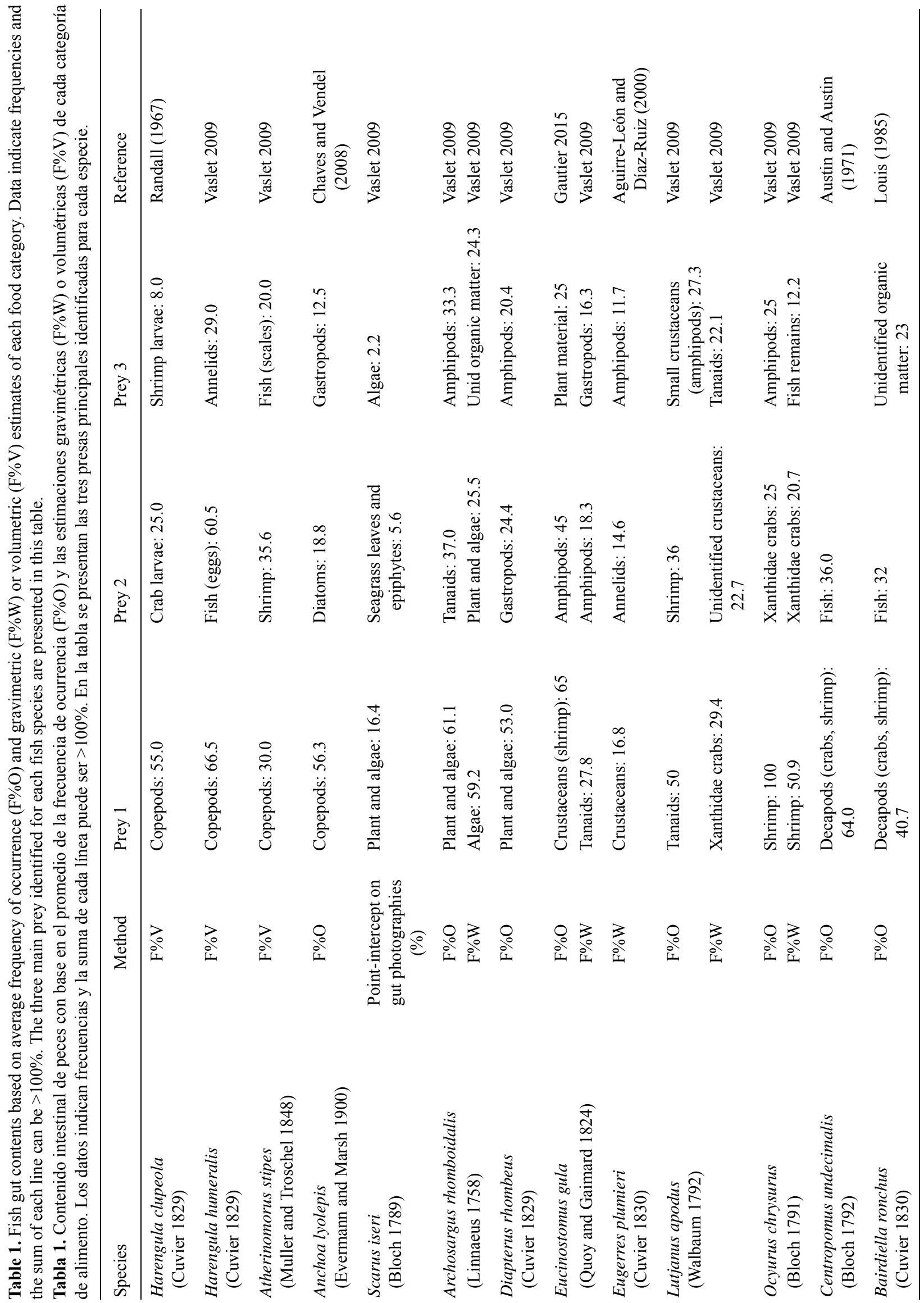




\section{Stable isotope analyses and mixing models}

Muscle tissues of fish, decapods, and molluscs, and other whole prey were oven dried at $50^{\circ} \mathrm{C}$ and ground to powder. As carbonate can interfere with organic carbon values (Bosley and Wainright 1999), calcified samples (i.e., algae, crustaceans) were decalcified with $10 \% \mathrm{HCl}$. As acid treatments have been reported to affect $\delta^{15} \mathrm{~N}$ values, subsamples dedicated to $\delta^{15} \mathrm{~N}$ analyses were not acidified (Sweeting et al. 2006). Stable isotope analyses of carbon and nitrogen were performed with a mass spectrometer (Optima; Isoprime, UK) coupled to a CNS elemental analyser (NA1200, ThermoScientific, European Union) at MARE Center, Liege University (Belgium). Isotopic ratios of carbon $\left(\delta^{13} \mathrm{C}\right)$ and nitrogen $\left(\delta^{15} \mathrm{~N}\right)$ were expressed as the relative per mil (\%o) difference between samples and standards (Vienna PDB for carbon, atmospheric $\mathrm{N}$ for nitrogen). Average reproductibilities based on replicate measurements were $0.19 \%$ for $\delta^{13} \mathrm{C}$ and $0.15 \%$ for $\delta^{15} \mathrm{~N}$. Variation in lipid content among organisms can affect the $\delta^{13} \mathrm{C}$ values and consequently the ecological interpretations (Post et al. 2007). Thus, the mathematical normalization outlined by Post et al. (2007) for aquatic organisms was used to standardize for lipid content: $\delta^{13} \mathrm{C}_{\text {normalized }}=\delta^{13} \mathrm{C}_{\text {untreated }}-3.32+0.99 \times \mathrm{C}: \mathrm{N}$. Normalization of $\delta^{13} \mathrm{C}$ values was based on $\mathrm{C}: \mathrm{N}$ values (i.e., $\mathrm{C}: \mathrm{N}$ ratios $>3.5$ for lipid-rich tissue) and they were calculated for gastropods, amphipods, annelids, and Majidae crabs (table 2).

The SIAR (stable isotope analysis in R) mixing model was used to determine the relative contribution of prey from mangroves and seagrass beds to the fish diets (Parnell et al. 2010). This model has the advantage of taking into account uncertainties associated with isotopic values of consumers and prey and trophic enrichment (Parnell et al. 2010). The SIAR mixing model allows considering the relative contribution of groups of prey a posteriori. In this model, fish isotopic values were corrected for trophic enrichment: $\Delta \mathrm{C}=1.0 \pm 0.3 \%$ and $\Delta \mathrm{N}=2.2 \pm 0.3 \%$ o for planktivores, herbivores, and omnivores, and $\Delta \mathrm{C}=0.5 \pm 0.1 \%$ and $\Delta \mathrm{N}=$ $3.4 \pm 0.2 \%$ for carnivores (Vander Zanden and Rasmussen 2001, McCutchan et al. 2003).

\section{Statistical analyses}

Statistical differences in $\delta^{13} \mathrm{C}$ and $\delta^{15} \mathrm{~N}$ values were tested using nonparametric tests (Mann-Whitney and Kruskal-Wallis tests) to overcome assumptions for normality and homogeneity of variance (Legendre and Legendre 1998). Spearman rank correlation coefficients between $\delta^{15} \mathrm{~N}$ and total length were computed using data from fish species in order to observe whether ontogenetic changes in diet could be detected. A significance level of $P \leq 0.05$ was used in all analyses. Statistical analyses were performed using XLSTAT and the SIAR package was run in R2.15.0 (R Development Core Team 2012).
1994, Nagelkerken et al. 2006, Chiaverini 2008): herbívoros que consumen algas y hojas de pastos marinos y sus algas epifitas; omnívoros que consumen invertebrados y por lo menos $10 \%$ de algas; planctívoros que consumen zooplancton; y carnívoros que consumen invertebrados bentónicos y algunas especies de peces.

En los manglares y pastos marinos se recolectaron muestras de las siguientes fuentes de alimento de los peces: hojarasca de manglar, hojas de pastos marinos, epifitas raspadas de las hojas de pastos marinos, anélidos, gasterópodos, anfípodos, cangrejos y camarones. Se recolectaron muestras de seston sobre las praderas de pastos marinos y de 10 a $20 \mathrm{~m}$ de los bordes del manglar mediante el arrastre de una red de plancton (100 $\mu \mathrm{m}$ de tamaño de malla) a $0.5 \mathrm{~m}$ por debajo de la superficie del agua durante $5 \mathrm{~min}$. En el laboratorio, el zooplancton (dominado por copépodos) se separó de la materia muerta suspendida.

\section{Análisis de isótopos estables y modelos de mezcla}

El tejido muscular de peces, decápodos y moluscos $\mathrm{y}$ otras presas enteras se secaron en un horno a $50{ }^{\circ} \mathrm{C}$ y se pulverizaron. Ya que el carbonato puede interferir con los valores del carbono orgánico (Bosley y Wainright 1999), las muestras calcificadas (i.e., algas, crustáceos) fueron decalcificadas con $\mathrm{HCl}$ al $10 \%$. En vista de que se ha documentado que los tratamientos con ácido afectan los valores de $\delta^{15} \mathrm{~N}$, no se acidificaron las submuestras dedicadas al análisis de $\delta^{15} \mathrm{~N}$ (Sweeting et al. 2006). El análisis de isótopos estables del carbono y el nitrógeno se realizó con un espectrómetro de masas (Optima; Isoprime, RU) junto con un analizador elemental CNS (NA1200, ThermoScientific, Unión Europea) en el Centro MARE de la Universidad de Lieja (Bélgica). Las razones isotópicas de carbono $\left(\delta^{13} \mathrm{C}\right)$ y nitrógeno $\left(\delta^{15} \mathrm{~N}\right)$ se expresaron como la diferencia en partes por mil (\%o) entre las muestras y los estándares (Viena PDB para carbono y $\mathrm{N}$ atmosférico para nitrógeno). La reproductibilidad promedio con base en mediciones replicadas fue de $0.19 \%$ para $\delta^{13} \mathrm{C} \mathrm{y}$ $0.15 \%$ para $\delta^{15} \mathrm{~N}$. Una variación en el contenido de lípidos entre organismos puede afectar los valores de $\delta^{13} \mathrm{C}$, consecuentemente, las interpretaciones ecológicas (Post et al. 2007). Por lo tanto, se usó la normalización matemática propuesta por Post et al. (2007) para organismos acuáticos para estandarizar el contenido lipídico: $\delta^{13} \mathrm{C}_{\text {normalizado }}=\delta^{13} \mathrm{C}_{\text {no tratado }}-$ $3.32+0.99 \times \mathrm{C}: \mathrm{N}$. La normalización de los valores de $\delta^{13} \mathrm{C}$ se basó en los valores de $\mathrm{C}: \mathrm{N}$ (i.e., razones $\mathrm{C}: \mathrm{N}>3.5$ para tejido rico en lípidos) y se calcularon para gasterópodos, anfípodos, anélidos y cangrejos de la familia Majidae (tabla 2).

Se usó el modelo de mezcla SIAR (análisis de isótopos estables en R) para determinar la contribución relativa de las presas procedentes de los manglares y pastos marinos a la dieta de los peces (Parnell et al. 2010). Este modelo tiene la ventaja de que toma en cuenta las incertidumbres asociadas con los valores isotópicos de consumidores y presas y el enriquecimiento trófico (Parnell et al. 2010). El modelo SIAR 
Table 2. Mean $\delta^{13} \mathrm{C}$ and $\delta^{15} \mathrm{~N}$ values ( \pm standard deviation) and $\mathrm{C}: \mathrm{N}$ ratios of prey items sampled in mangroves and seagrass beds ( $n$, number of samples). See figure 1 for location of sampling sites.

Tabla 2. Valores medios de $\delta^{13} \mathrm{C}$ y $\delta^{15} \mathrm{~N}$ ( \pm desviación estándar) y razones C: $\mathrm{N}$ de las fuentes de alimento de peces provenientes de manglares y pastos marinos ( $n$, número de muestras). Ver la figura 1 para la ubicación de los sitios de muestreo.

\begin{tabular}{|c|c|c|c|c|}
\hline Sample & $n$ & $\delta^{13} \mathrm{C}(\%)$ & $\delta^{15} \mathrm{~N}(\%)$ & $\mathrm{C}: \mathrm{N}$ \\
\hline \multicolumn{5}{|c|}{ Offshore mangrove islet: Fajou islet (site F) } \\
\hline Mangrove litter & 2 & $-30.0 \pm 0.2$ & $-1.3 \pm 0.3$ & 212.7 \\
\hline \multicolumn{5}{|l|}{ Algae } \\
\hline Rhodophyceae: Spyridia filamentosa & 3 & $-27.8 \pm 0.6$ & $-0.2 \pm 0.2$ & 58.7 \\
\hline \multicolumn{5}{|l|}{ Molluscs: Gastropods } \\
\hline Littorinidae: Littoraria cf nebulosa & 6 & $-22.4 \pm 1.1$ & $0.03 \pm 1.2$ & 4.2 \\
\hline Amphipods & 5 & $-17.0 \pm 0.5$ & $4.8 \pm 0.2$ & 2.8 \\
\hline \multicolumn{5}{|l|}{ Decapods } \\
\hline Palaemonidae: Palemon cf northropi & 5 & $-18.6 \pm 1.8$ & $6.1 \pm 0.2$ & 3.2 \\
\hline Zooplankton & 3 & $-20.1 \pm 0.3$ & $7.6 \pm 0.2$ & 4.8 \\
\hline Annelids: Polychaetes & 3 & $-16.8 \pm 0.9$ & $6.2 \pm 1.1$ & 3.9 \\
\hline \multicolumn{5}{|l|}{ Molluscs: Gastropods } \\
\hline Littorinidae: Littoraria cf nebulosa & 6 & $-22.1 \pm 1.1$ & $0.05 \pm 1.9$ & 4.1 \\
\hline Amphipods & 4 & $-19.6 \pm 0.8$ & $4.3 \pm 0.4$ & 2.5 \\
\hline \multicolumn{5}{|l|}{ Decapods } \\
\hline Xanthidae: Eurytium limosum & 3 & $-15.8 \pm 0.3$ & $8.3 \pm 0.06$ & 3.4 \\
\hline Porcellanidae: Petrolisthes armatus & 4 & $-16.0 \pm 0.1$ & $6.4 \pm 0.1$ & 3.4 \\
\hline Portunidae: Callinectes sapidus & 3 & $-23.3 \pm 1.1$ & $5.8 \pm 0.3$ & 3.2 \\
\hline Pheophyceae: Dictyota cf pulchella & 6 & $-15.9 \pm 0.2$ & $5.2 \pm 0.6$ & 18.3 \\
\hline \multicolumn{5}{|l|}{ Seagrass leaves } \\
\hline Thalassia testudinum & 7 & $-10.4 \pm 0.2$ & $2.8 \pm 1.1$ & 18.9 \\
\hline Syringodium filiforme & 4 & $-10.9 \pm 0.4$ & $3.0 \pm 0.6$ & 23.0 \\
\hline Epiphytes from seagrass leaves & 6 & $-11.6 \pm 0.5$ & $2.2 \pm 0.3$ & 24.1 \\
\hline Annelids: Polychaetes & 3 & $-12.7 \pm 0.2$ & $6.3 \pm 0.1$ & 4.4 \\
\hline \multicolumn{5}{|l|}{ Molluscs: Gastropods } \\
\hline Cerithidae: Cerithium litteratum & 5 & $-10.5 \pm 0.9$ & $6.7 \pm 0.1$ & 3.6 \\
\hline Amphipods & 6 & $-14.4 \pm 0.2$ & $6.3 \pm 0.1$ & 4.4 \\
\hline \multicolumn{5}{|l|}{ Decapods } \\
\hline Penaeidae: Penaeus cf similis & 6 & $-14.0 \pm 0.7$ & $7.2 \pm 0.1$ & 3.1 \\
\hline Xanthidae & 6 & $-12.2 \pm 0.9$ & $7.7 \pm 0.8$ & 3.6 \\
\hline Portunidae: Portunus sebae & 3 & $-13.2 \pm 0.1$ & $5.8 \pm 0.1$ & 3.4 \\
\hline
\end{tabular}




\section{RESUlTS}

The prey collected from magroves were more depleted in ${ }^{13} \mathrm{C}\left(\delta^{13} \mathrm{C}_{\text {mean }}=-20.4 \pm 1.2 \%\right.$ o $)$ than those collected from seagrass beds $\left(\delta^{13} \mathrm{C}_{\text {mean }}=-12.7 \pm 0.5 \%\right.$ ) (Mann-Whitney test, $P<0.001$ ) (table 2; figs. 2, 3). Mangrove invertebrates presented $\delta^{13} \mathrm{C}$ values varying between $-25.1 \%$ (Littorinidae gastropods) and $-15.5 \%$ (amphipods) (table 2; figs. 2, 3). On average, the mangrove invertebrates that were the most depleted in ${ }^{13} \mathrm{C}$ were Portunidae crabs and Littorinidae gastropods (mean $\delta^{13} \mathrm{C}$ values of $-23.3 \%$ and $-22.3 \%$, respectively; table 2 ).

Mean $\delta^{15} \mathrm{~N}$ values were lowest for primary producers, such as mangrove litter, algae, seagrasses, and epiphytic algae ( $-1.5 \%$ o to $5.2 \%$ ) ; intermediate $(4.5 \%$ o to $6.3 \%$ ) for amphipods; and higher (5.8\% to $8.3 \%$ ) for other invertebrates, such as zooplankton, annelids, molluscs, and decapods (table 2). Littorinidae gastropods from mangroves were characterized by much lower mean $\delta^{15} \mathrm{~N}$ values $(0.04 \%$ ) compared to other invertebrates (table 2). Elemental ratios $(\mathrm{C}: \mathrm{N}, \mathrm{w}: \mathrm{w})$, which are indicative of prey nutritional values in terms of digestibility, presented lower values in animal food sources $\left(C: \mathrm{N}_{\text {mean }}=3.9 \pm 0.1\right)$ than in plant items $\left(C: \mathrm{N}_{\text {mean }}=\right.$ $43.3 \pm 16.3)$, testifying the high nutritive quality of the former (Mann-Whitney test, $P<0.001$ ) (table 2).

Mean $\delta^{13} \mathrm{C}$ values for the 12 transient fish species collected in mangroves and seagrass beds ranged from $-18.7 \pm$ $1.2 \%$ for the snook Centropomus undecimalis (site G) to $-12.0 \pm 0.3 \%$ o for the snapper Lutjanus apodus (site S) (table 3). On average, fish species collected in seagrass beds (site $\mathrm{S}$ ) were more enriched in ${ }^{13} \mathrm{C}\left(\delta^{13} \mathrm{C}_{\text {mean }}=-13.0 \pm 0.3 \%\right.$ ) than species from the mangrove sites (sites $\mathrm{F}$ and $\mathrm{G},-14.9 \pm$ $0.4 \%$ and $-14.7 \pm 0.5 \%$, respectively) (table 3 ). The $\delta^{15} \mathrm{~N}$ values were lower for herbivores; intermediate for planktivores, omnivores, and carnivores foraging exclusively on invertebrates; and higher for carnivores consuming both invertebrates and fishes (table 3 ).

As $\delta^{13} \mathrm{C}$ values significantly differed between food sources from mangroves and seagrass beds, the relative importance of these prey items in the fish diets could be assessed. Three out of 12 fish species had depleted $\delta^{13} \mathrm{C}$ values that are closer to those of mangrove prey: the planktivores Anchoa lyolepis (sites F, G) and Harengula clupeola (sites F, G), and the carnivore C. undecimalis (site G). Specimens of L. apodus from all sites had $\mathrm{C}$ signatures closer to those of prey from seagrass beds (fig. 3). The values of both $\delta^{13} \mathrm{C}$ and $\delta^{15} \mathrm{~N}$ for Ocyurus chrysurus increased between small $(6 \mathrm{~cm})$ and large specimens $(12 \mathrm{~cm}$; Spearman rank correlation, $P<0.0001$ ) (table 3; fig. 3b), suggesting different diets for this fish species depending on specimen sizes (fig. 3).

The SIAR mixing models showed that the two planktivores $A$. lyolepis and $H$. clupeola presented higher mean contributions (95\% Bayesian confidence intervals [BCI]) of mangrove food sources in their diets (78-83\%) than the considerar la contribución relativa de grupos de presas $a$ posteriori. En este modelo, se corrigieron los valores isotópicos de los peces para enriquecimiento trófico: $\Delta \mathrm{C}=1.0 \pm$ $0.3 \%$ y $\Delta \mathrm{N}=2.2 \pm 0.3 \%$ para planctívoros, herbívoros y omnívoros, y $\Delta \mathrm{C}=0.5 \pm 0.1 \%$ y $\Delta \mathrm{N}=3.4 \pm 0.2 \%$ para carnívoros (Vander Zanden y Rasmussen 2001, McCutchan et al. 2003).

\section{Análisis estadísticos}

Se determinaron las diferencias estadísticas entre los valores de $\delta^{13} \mathrm{C}$ y $\delta^{15} \mathrm{~N}$ mediante pruebas no paramétricas (pruebas de Mann-Whitney y Kruskal-Wallis) para comprobar los supuestos de normalidad y homogeneidad de varianza (Legendre y Legendre 1998). Se calcularon los coeficientes de correlación por rangos de Spearman entre $\delta^{15} \mathrm{~N}$ y la longitud total con datos de las especies de peces para determinar si se podían detectar cambios ontogenéticos en la dieta. Se usó un nivel de significación de $P \leq 0.05$ en todos los análisis. Los análisis estadísticos se realizaron con XL-STAT y el programa SIAR se corrió en R2.15.0 (R Development Core Team 2012).

\section{RESULTADOS}

Las presas provenientes de los manglares presentaron valores más empobrecidos en ${ }^{13} \mathrm{C}\left(\delta^{13} \mathrm{C}_{\text {media }}=-20.4 \pm 1.2 \%\right.$ o que las presas de los pastos marinos $\left(\delta^{13} \mathrm{C}_{\text {media }}=-12.7 \pm\right.$ $0.5 \%$ ) (prueba de Mann-Whitney, $P<0.001$ ) (tabla 2; figs. 2, 3). Los valores de $\delta^{13} \mathrm{C}$ de los invertebrados recolectados en los manglares variaron entre $-25.1 \%$ (gasterópodos de Littorinidae) y -15.5\% (anfípodos) (tabla 2; figs. 2, 3). En promedio, los invertebrados de los manglares más empobrecidos en ${ }^{13} \mathrm{C}$ fueron cangrejos de la familia Portunidae $\mathrm{y}$ gasterópodos de la familia Littorinidae (valores medios de $\delta^{13} \mathrm{C}$ de $-23.3 \%$ y $-22.3 \%$, respectivamente; tabla 2 ).

Los valores medios de $\delta^{15} \mathrm{~N}$ fueron menores $(-1.5 \%$ a $5.2 \%$ ) para los productores primarios como hojarasca de manglar, algas, pastos marinos y algas epifitas; intermedios (4.5\% a 6.3\%o) para anfípodos; y mayores (5.8\% a $8.3 \%$ ) para otros invertebrados como zooplancton, anélidos, molúscos y decápodos (tabla 2). Los valores medios de $\delta^{15} \mathrm{~N}$ para los gasterópodos de la familia Littorinidae (0.04\%o) fueron mucho menores que los de otros invertebrados (tabla 2). Las razones elementales ( $\mathrm{C}: \mathrm{N}, \mathrm{p}: \mathrm{p})$, las cuales son indicativas del valor nutricional de las presas en cuanto a digestibilidad, fueron menores para alimentos de origen animal $\left(\mathrm{C}: \mathrm{N}_{\text {media }}=3.9 \pm 0.1\right)$ que para componentes vegetales $\left(\mathrm{C}: \mathrm{N}_{\text {media }}=43.3 \pm 16.3\right)$, lo que demuestra la alta calidad nutritiva de los primeros (prueba de Mann-Whitney, $P<$ 0.001) (tabla 2).

Los valores medios de $\delta^{13} \mathrm{C}$ para las 12 especies de peces transeúntes capturadas en los manglares y pastos marinos variaron entre $-18.7 \pm 1.2 \%$ para el róbalo común Centropomus undecimalis (sitio G) y $-12.0 \pm 0.3 \%$ para 
Vaslet et al.: Foraging habits of juvenile fishes in a Caribbean lagoon

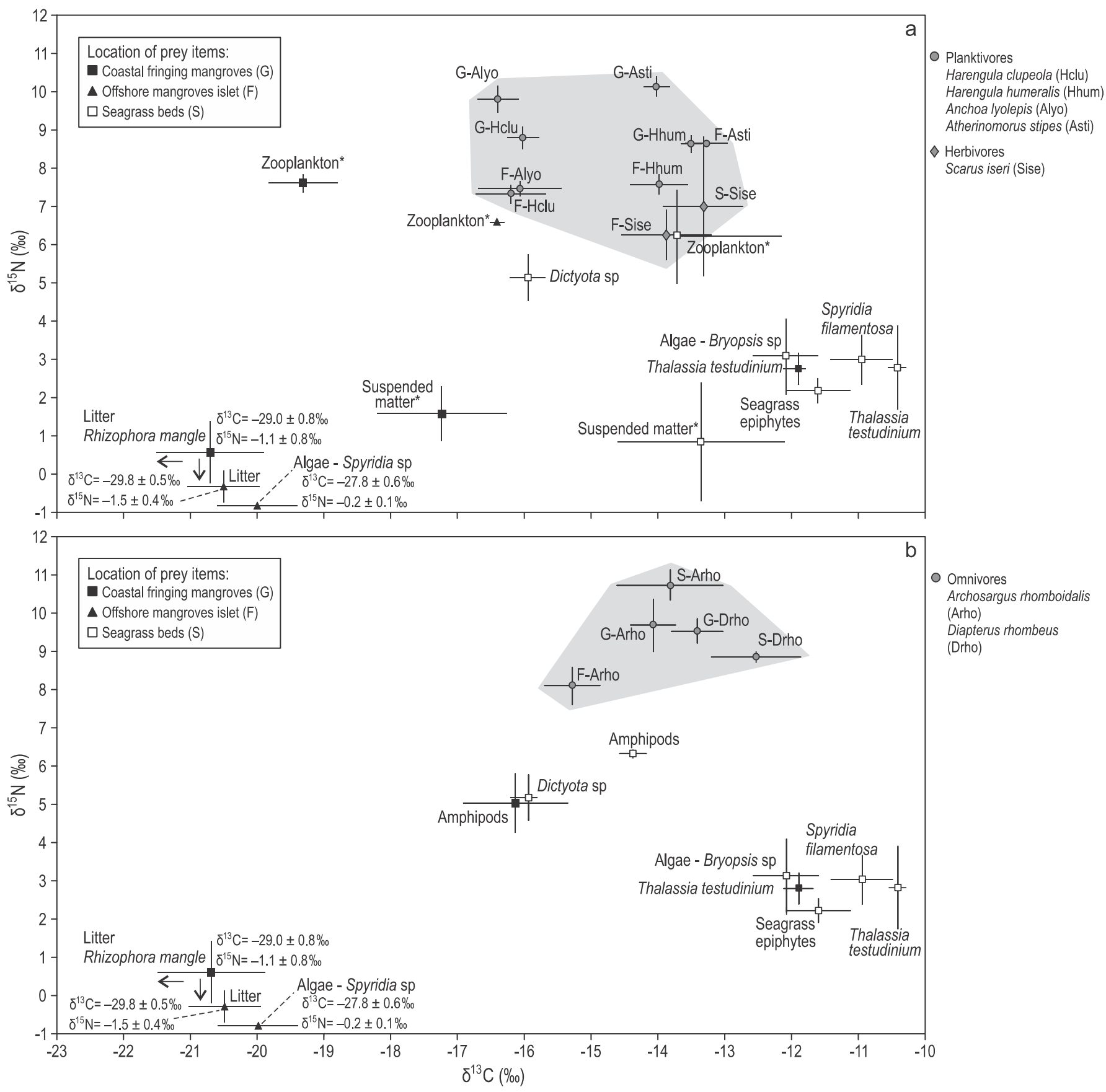

Figure 2. Mean $\delta^{13} \mathrm{C}$ and $\delta^{15} \mathrm{~N}$ values ( \pm standard deviation) of (a) planktivorous and herbivorous fishes and (b) omnivorous fishes (grey areas) and their prey sampled in mangroves and seagrass beds. The asterisks indicate potential prey of planktivorous fishes.

Figura 2. Valores medios ( \pm desviación estándar) de $\delta^{13} \mathrm{C}$ y $\delta^{15} \mathrm{~N}$ de (a) peces planctívoros y herbívoros y (b) peces omnívoros (zonas grises) y sus presas provenientes de manglares y pastos marinos. Los asteriscos indican las presas potenciales de los peces planctívoros. 


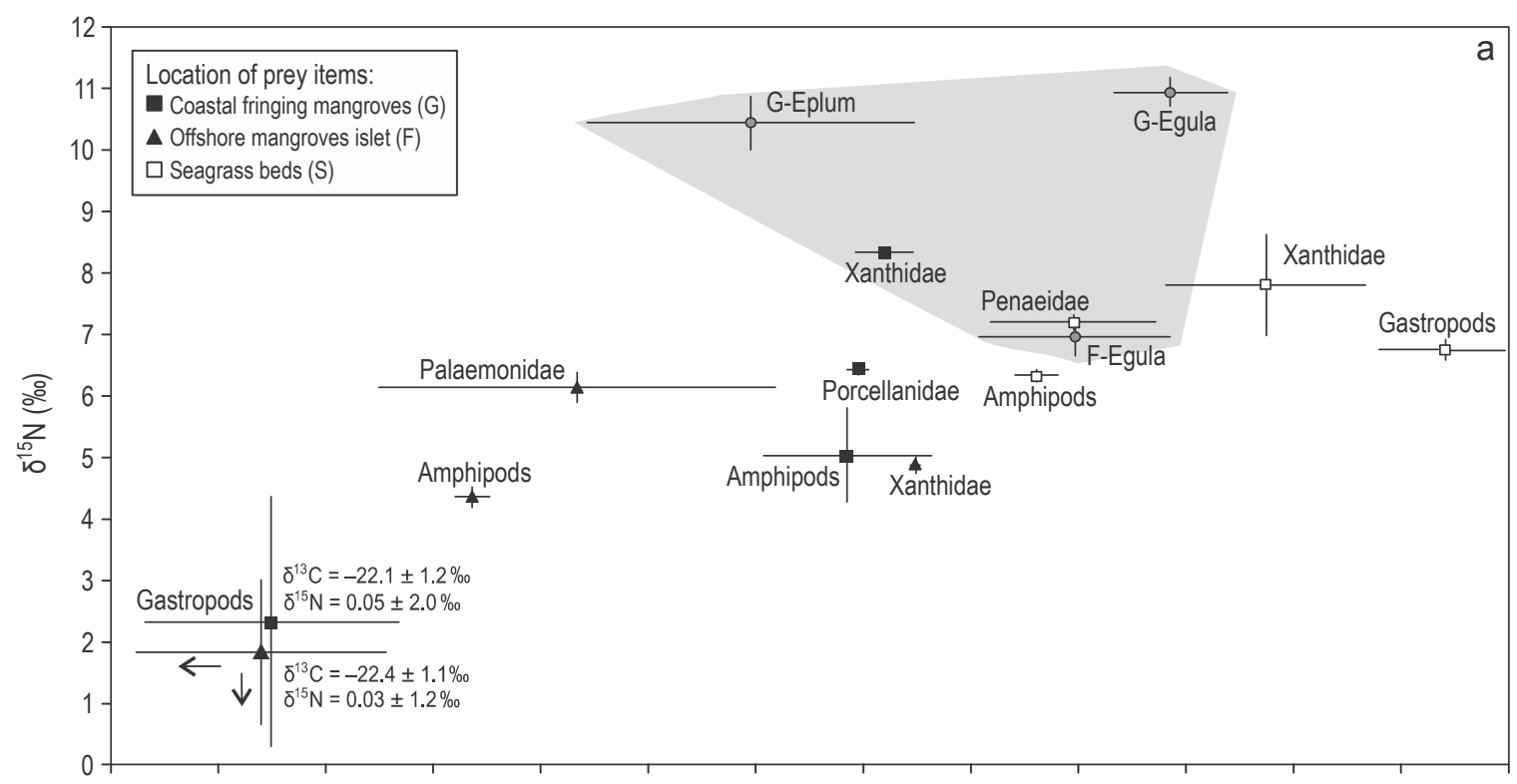

Carnivores

Eucinostomus gula

Eugerres plumieri

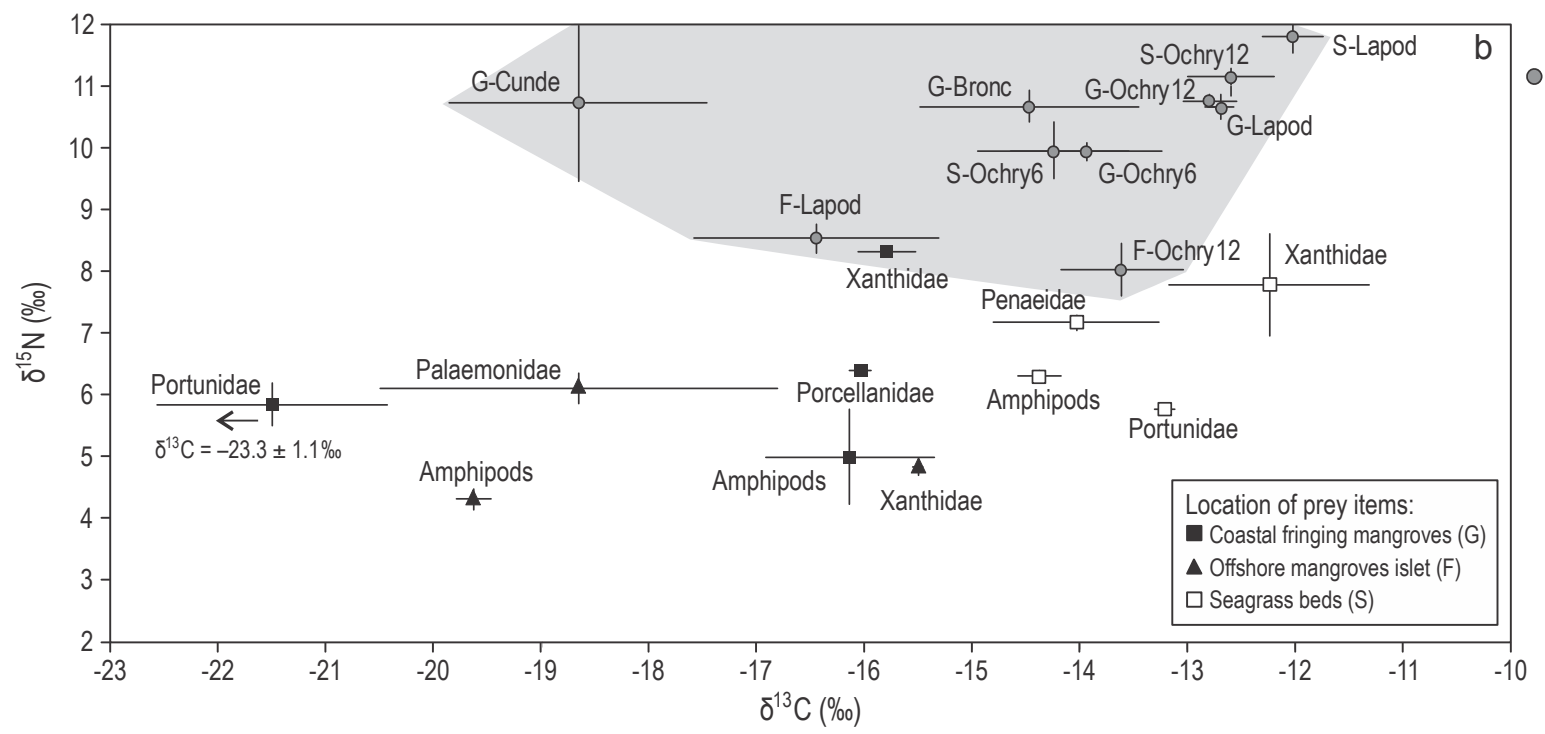

Carnivores

Lutjanus apodus Ocyurus chrysurus Centropomus undecimalis Bairdiella ronchus

Figure 3. Mean $\delta^{13} \mathrm{C}$ and $\delta^{15} \mathrm{~N}$ values ( \pm standard deviation) of the carnivorous fishes (a) Eugerres plumieri and Eucinostomus gula and (b) Lutjanus apodus, Ocyurus chrysurus, Centropomus undecimalis, and Bairdiella ronchus (grey areas) and their prey items sampled in mangroves and seagrass beds.

Figura 3. Valores medios ( \pm desviación estándar) de $\delta^{13} \mathrm{C}$ y $\delta^{15} \mathrm{~N}$ de los peces carnívoros (a) Eugerres plumieri y Eucinostomus gula y (b) Lutjanus apodus, Ocyurus chrysurus, Centropomus undecimalis y Bairdiella ronchus (zonas grises) y sus presas provenientes de manglares y pastos marinos.

other two planktivorous species, Harengula humeralis and Atherinomorus stipes, which relied equally on mangrove and seagrass bed food sources (table 4). The carnivores C. undecimalis and the mangrove resident fish species B. ronchus presented mangrove prey mean contributions (95\% BCI) of $61.3 \%(40.7-83.1 \%)$ and $42.3 \%$ (29.3-55.3\%), respectively (table 4). In contrast, the herbivore Scarus iseri, the omnivores Archosargus rhomboidalis and Diapterus rhombeus, and the carnivores Eucinostomus gula, L. apodus, and the large-sized juveniles of $O$. chrysurus relied more on el pargo amarillo Lutjanus apodus (sitio S) (tabla 3). En promedio, las especies de peces recolectadas en los pastos marinos (sitio $\mathrm{S}$ ) presentaron mayor enriquecimiento en ${ }^{13} \mathrm{C}$ $\left(\delta^{13} \mathrm{C}_{\text {media }}=-13.0 \pm 0.3 \%\right.$ ) que las especies provenientes de los manglares (sitios F y G, $-14.9 \pm 0.4 \%$ y $-14.7 \pm 0.5 \%$, respectivamente) (tabla 3). Los valores de $\delta^{15} \mathrm{~N}$ fueron menores para los herbívoros; intermedios para los planctívoros, omnívoros y carnívoros que se alimentan exclusivamente de invertebrados; y mayores para los carnívoros que consumen tanto invertebrados como peces (tabla 3 ). 
Table 3. Mean $\delta^{13} \mathrm{C}$ and $\delta^{15} \mathrm{~N}$ values ( \pm standard deviation) and $\mathrm{C}: \mathrm{N}$ ratios of fish species sampled in mangroves and seagrass beds (see fig. 1 for location). Abbreviations: $n$, number of samples; TL, total length; A/J, adult or juvenile specimens. Fish diets are indicated after the name of the species: P, plant material; Z, zooplankton; I, invertebrates; F, fish.

Tabla 3. Valores medios de $\delta^{13} \mathrm{C}$ y $\delta^{15} \mathrm{~N}$ ( \pm desviación estándar) y razones C:N de las especies de peces recolectadas en manglares y pastos marinos (ver fig. 1 para su ubicación). Abreviaciones: $n$, número de muestras; TL, longitud total; A/J, especímenes adultos o juveniles. La dieta de los peces se muestra después del nombre de la especie: P, material vegetal; Z, zooplancton; I, invertebrados; F, peces.

\begin{tabular}{|c|c|c|c|c|c|c|}
\hline Fish species & $n$ & $\mathrm{TL}(\mathrm{cm})$ & $\mathrm{A} / \mathrm{J}$ & $\delta^{13} \mathrm{C}(\%)$ & $\delta^{15} \mathrm{~N}(\%)$ & $\mathrm{C}: \mathrm{N}$ \\
\hline Harengula clupeola $(\mathrm{Z})$ & 6 & $4-9$ & $\mathrm{~J}$ & $-16.2 \pm 0.5$ & $7.3 \pm 0.2$ & 3.2 \\
\hline Harengula humeralis $(\mathrm{Z})$ & 4 & $6-7$ & $\mathrm{~J}$ & $-13.9 \pm 0.4$ & $7.6 \pm 0.2$ & 3.2 \\
\hline Anchoa lyolepis (Z) & 6 & 8 & $\mathrm{~J}$ & $-16.0 \pm 0.6$ & $7.5 \pm 0.2$ & 3.3 \\
\hline \multicolumn{7}{|l|}{ Herbivores } \\
\hline Scarus iseri $(\mathrm{P})$ & 6 & $4-7$ & $\mathrm{~J}$ & $-13.8 \pm 0.7$ & $6.3 \pm 0.6$ & 3.2 \\
\hline \multicolumn{7}{|l|}{ Omnivores } \\
\hline Archosargus rhomboidalis (P, I) & 6 & $10-17$ & A & $-15.3 \pm 0.4$ & $8.1 \pm 0.5$ & 3.1 \\
\hline \multicolumn{7}{|l|}{ Carnivores } \\
\hline
\end{tabular}

Coastal mangroves: Grande Rivière à Goyaves (site G)

\begin{tabular}{|c|c|c|c|c|c|c|}
\hline \multicolumn{7}{|l|}{ Planktivores } \\
\hline Harengula clupeola $(\mathrm{Z})$ & 6 & 9 & $\mathrm{~J}$ & $-16.0 \pm 0.2$ & $8.8 \pm 0.3$ & 3.2 \\
\hline Harengula humeralis (Z) & 6 & 8 & $\mathrm{~J}$ & $-13.5 \pm 0.1$ & $8.6 \pm 0.2$ & 3.2 \\
\hline Anchoa lyolepis (Z) & 6 & 8 & $\mathrm{~J}$ & $-16.4 \pm 0.3$ & $9.8 \pm 0.3$ & 3.1 \\
\hline Atherinomorus stipes & 6 & 6.5 & A & $-14.0 \pm 0.2$ & $10.1 \pm 0.2$ & 3.2 \\
\hline \multicolumn{7}{|l|}{ Omnivores } \\
\hline Archosargus rhomboidalis (P, I) & 6 & $10-14$ & A & $-14.1 \pm 0.3$ & $9.7 \pm 0.7$ & 3.1 \\
\hline \multicolumn{7}{|l|}{ Carnivores } \\
\hline Eucinostomus gula (I) & 6 & $8-10$ & A & $-13.1 \pm 0.5$ & $10.9 \pm 0.2$ & 3.2 \\
\hline Eugerres plumieri (I) & 6 & $25-32$ & A & $-17.0 \pm 1.5$ & $10.4 \pm 0.4$ & 3.2 \\
\hline Lutjanus apodus (I, F) & 6 & $8-9$ & $\mathrm{~J}$ & $-12.7 \pm 0.1$ & $10.6 \pm 0.2$ & 3.2 \\
\hline Ocyurus chrysurus (I) & 6 & 6 & $\mathrm{~J}$ & $-13.9 \pm 0.7$ & $9.9 \pm 0.1$ & 3.2 \\
\hline \multicolumn{7}{|c|}{ Seagrass beds (site $S$ ) } \\
\hline \multicolumn{7}{|l|}{ Herbivores } \\
\hline Scarus iseri $(\mathrm{P})$ & 6 & 7 & $\mathrm{~J}$ & $-13.3 \pm 0.6$ & $7.0 \pm 1.8$ & 0.9 \\
\hline \multicolumn{7}{|l|}{ Omnivores } \\
\hline Archosargus rhomboidalis (P, I) & 6 & $11-14$ & A & $-13.8 \pm 0.8$ & $10.7 \pm 0.4$ & 2.6 \\
\hline Diapterus rhombeus (P, I) & 3 & 13 & $\mathrm{~J}$ & $-12.5 \pm 0.6$ & $8.8 \pm 0.1$ & 3.1 \\
\hline \multicolumn{7}{|l|}{ Carnivores } \\
\hline Eucinostomus gula (I) & 6 & 10 & A & $-12.2 \pm 0.2$ & $11.2 \pm 0.2$ & 3.1 \\
\hline Lutjanus apodus (I, F) & 6 & $8-14$ & $\mathrm{~J}$ & $-12.0 \pm 0.3$ & $11.7 \pm 0.2$ & 3.1 \\
\hline Ocyurus chrysurus (I) & 6 & $4-6$ & $\mathrm{~J}$ & $-14.2 \pm 0.7$ & $9.9 \pm 0.4$ & 3.2 \\
\hline
\end{tabular}


seagrass prey than on mangrove prey, regardless of the sampling site (table 4). However, the low contribution percentages obtained for the omnivores and the resident carnivore $B$. ronchus indicated that some additional prey were missing in the model. A slight increase in the proportion of seagrass prey items in the diet of $O$. chrysurus was observed from small specimens $(95 \%$ BCI of seagrass prey varying between $29.6 \%$ and $62.1 \%$ ) to larger ones $(95 \%$ BCI of seagrass prey varying between $29.1 \%$ and $85.6 \%$ ) (table 4 ).

\section{Discussion}

Prey from mangroves are more depleted in ${ }^{13} \mathrm{C}$ compared to those from seagrass beds (Kieckbusch et al. 2004, Nagelkerken et al. 2006, Lugendo et al. 2007), which allows assessing their relative importance in fish diets. The variations in carbon isotope values is due to different photosynthetic pathways and the different isotopic compositions in inorganic carbon used by primary producers (i.e., mangroves and seagrass beds) (Hemminga and Mateo 1996, Raven et al. 2002, Bouillon et al. 2008). This depletion in ${ }^{13} \mathrm{C}$ is also observed for some invertebrates (e.g., annelids, amphipods, crabs) suggesting that they forage on mangrove resources (Christensen et al. 2001, Bouillon et al. 2002, Nagelkerken and van der Velde 2004b). Mangrove Littorinidae are characterized by low nitrogen values (Christensen et al. 2001, this study). This pattern could be due to the consumption of emerged epiphytes particularly depleted in ${ }^{15} \mathrm{~N}$ (Christensen et al. 2001, Bouillon et al. 2004). These epiphytes incorporate depleted nitrogen sources, such as $\mathrm{N}_{2}$ or volatilized ammonia coming from mangrove material remineralization (Bouillon et al. 2004, Fogel et al. 2008).

Two groups of transient fishes were distinguished in GCSM according to their $\delta^{13} \mathrm{C}$ : most of the fishes had enriched carbon values close to those of seagrass food sources and only a few specimens of transient fishes had relatively depleted carbon values, suggesting that these specimens complemented their diets with mangrove prey items. Mangrove food resources were consumed by mangrove resident species, such as $B$. ronchus, or by species that tolerate freshwater inputs and turbid waters, such as $C$. undecimalis (Louis 1985, Aguirre-León and Díaz-Ruiz 2000). Nutrient inputs and the abundance of mangrove infauna can explain the importance of mangrove prey in the diet of some transient fishes (Rodelli et al. 1984, Lugendo et al. 2007, Thollot et al. 1999).

For most juvenile transient fishes, seagrass beds represented their main feeding habitat even if the fishes sheltered in mangroves. These results concur with findings from studies performed in the Caribbean region highlighting that juvenile parrotfishes (Scaridae) shelter in mangroves and forage on epiphytic algae growing on seagrass leaves (Moncreiff and Sullivan 2001, Cocheret de la Morinière et al. 2003a, Nagelkerken et al. 2006). This feeding behavior could be due to high nutritional value and high productivity of epiphytic
Como los valores de $\delta^{13} \mathrm{C}$ difirieron significativamente entre las fuentes de alimento provenientes de los manglares y pastos marinos, fue posible evaluar la importancia relativa de estos componentes en la dieta de los peces. Tres de las 12 especies de peces presentaron valores de $\delta^{13} \mathrm{C}$ empobrecidos que se asemejan más a los de las presas de los manglares: los planctívoros Anchoa lyolepis (sitios F, G) y Harengula clupeola (sitios F, G), y el carnívoro C. undecimalis (sitio G). Los especímenes de L. apodus de los tres sitios presentaron huellas de $\mathrm{C}$ similares a los de las presas provenientes de los pastos marinos (fig. 3). Los valores de tanto $\delta^{13} \mathrm{C}$ como $\delta^{15} \mathrm{~N}$ para Ocyurus chrysurus aumentaron entre los especímenes pequeños $(6 \mathrm{~cm})$ y grandes $(12 \mathrm{~cm}$; correlación de Spearman, $P<0.0001$ ) (tabla 3; fig. 3b), lo que sugiere diferentes dietas para esta especie según su tamaño (fig. 3).

El modelo de mezcla SIAR mostró que las contribuciones medias (intervalo de confianza bayesiano [ICB] al 95\%) de las fuentes de alimento provenientes de los manglares fueron mayores en las dietas de los planctívoros A. lyolepis y H. clupeola (78-83\%) que en las dietas de las otras dos especies de planctívoros, Harengula humeralis y Atherinomorus stipes, las cuales dependieron igualmente de las fuentes provenientes de los manglares y pastos marinos (tabla 4). Las contribuciones medias (ICB al 95\%) de las fuentes de alimento provenientes de los manglares a las dietas de los carnívoros C. undecimalis y B. ronchus (pez residente de los manglares) fueron $61.3 \%(40.7-83.1 \%)$ y $42.3 \%$ (29.3-55.3\%), respectivamente (tabla 4). En contraste, el herbívoro Scarus iseri, los omnívoros Archosargus rhomboidalis y Diapterus rhombeus, y los carnívoros Eucinostomus gula, L. apodus y los juveniles de talla grande de O. chrysurus dependieron más del alimento proveniente de los pastos marinos que de los manglares, independientemente del sitio de muestreo (tabla 4). No obstante, los bajos porcentajes de contribución obtenidos para los omnívoros y el carnívoro residente $B$. ronchus indicaron que faltaban algunas presas adicionales en el modelo. En la dieta de $O$. chrysurus se observó un ligero incremento en la proporción de componentes provenientes de los pastos marinos entre los especímenes pequeños (ICB al $95 \%$ de presas oscilando entre $29.6 \%$ y $62.1 \%$ ) y los de mayor tamaño (ICB al $95 \%$ de presas oscilando entre $29.1 \%$ y $85.6 \%$ ) (tabla 4 ).

\section{Discusión}

Las fuentes de alimento provenientes de los manglares están más empobrecidas en ${ }^{13} \mathrm{C}$ que las de praderas de pastos marinos (Kieckbusch et al. 2004, Nagelkerken et al. 2006, Lugendo et al. 2007), lo que permite evaluar su importancia relativa en las dietas de los peces. Las variaciones de los valores del isótopo de carbono se atribuyen a las diferentes rutas fotosintéticas y a la diferencia de la composición isotópica del carbono inorgánico usado por los productores primarios (i.e., manglares y pastos marinos) (Hemminga y Mateo 1996, Raven et al. 2002, Bouillon et al. 2008). 
Table 4. Mean contributions ( $95 \%$ Bayesian confidence intervals $[\mathrm{BCI}]$ ) of food sources from mangroves and seagrass beds in the fish diets. Bold numbers indicate the highest contributions of food sources. Sites: offshore mangrove islet (Fajou, F), coastal mangroves (Grande Rivière à Goyaves, G), and seagrass beds (S).

Tabla 4. Contribuciones medias (intervalos de confianza bayesianos [BCI] al 95\%) de las fuentes de alimento provenientes de manglares y pastos marinos en la dieta de los peces. Los números en negritas indican las mayores contribuciones de las fuentes de alimento. Sitios: islote de manglares (Fajou, F), manglares costeros (Grande Rivière à Goyaves, G) y praderas de pastos marinos (S).

\begin{tabular}{|c|c|c|c|c|}
\hline Fish species & Site & \multicolumn{3}{|c|}{ Mean (95 BCI) $(\%)$} \\
\hline \multicolumn{5}{|l|}{ Planktivores } \\
\hline \multirow[t]{2}{*}{ Harengula clupeola } & $\mathrm{F}$ & $80.4(67.7-94.4)$ & & $19.5(5.6-32.3)$ \\
\hline & $\mathrm{G}$ & & $80.1(68.6-91.2)$ & $19.8(8.0-31.3)$ \\
\hline Harengula humeralis & $\mathrm{F}$ & $48.2(32.4-63.4)$ & & $51.8(36.6-67.6)$ \\
\hline \multirow[t]{2}{*}{ Anchoa lyopelis } & $\mathrm{F}$ & $77.9(64.3-92.7)$ & & $22.0(7.3-35.7)$ \\
\hline & G & & $82.6(70.7-94.9)$ & $17.4(5.0-29.2)$ \\
\hline \multirow{2}{*}{ Atherinomorus stipes } & $\mathrm{F}$ & $45.3(22.9-69.3)$ & & $54.6(30.6-77.0)$ \\
\hline & G & & $51.5(33.9-67.5)$ & $48.4(32.4-66.0)$ \\
\hline \multicolumn{5}{|l|}{ Herbivores } \\
\hline \multirow[t]{3}{*}{ Archosargus rhomboidalis } & $\mathrm{G}$ & & $27.2(12.6-45.3)$ & $49.3(34.3-64.9)$ \\
\hline & $\mathrm{F}$ & $29.2(14.8-39.4)$ & & $40.9(26.5-55.3)$ \\
\hline & $\mathrm{S}$ & & $22.7(9.2-35.3)$ & $47.3(30.7-64.0)$ \\
\hline \multirow[t]{2}{*}{ Diapterus rhombeus } & G & $29.3(13.6-44.5)$ & & $46.2(30.2-62.4)$ \\
\hline & $\mathrm{S}$ & & $29.5(14.2-44.3)$ & $42.9(25.0-61.2)$ \\
\hline \multicolumn{5}{|l|}{ Carnivores } \\
\hline \multirow[t]{3}{*}{ Eucinostomus gula } & $\mathrm{G}$ & & $26.4(12.7-39.6$ & $52.8(38.8-67.0)$ \\
\hline & $\mathrm{F}$ & $31.9(18.0-45.4)$ & & $36.1(21.6-50.5)$ \\
\hline & $\mathrm{S}$ & & $17.4(4.9-30.2)$ & $60.8(38.2-80.1)$ \\
\hline \multirow{3}{*}{ Ocyurus chrysurus } & $\mathrm{G}: 12 \mathrm{~cm}$ & & $31.7(14.3-52.6)$ & $68.3(47.3-85.6)$ \\
\hline & $\mathrm{S}: 12 \mathrm{~cm}$ & & $36.9(15.1-63.3)$ & $63.0(36.7-84.9)$ \\
\hline & $\mathrm{F}: 12 \mathrm{~cm}$ & $50.5(30.1-70.9)$ & & $49.4(29.1-69.8)$ \\
\hline Bairdiella ronchus & $\mathrm{G}$ & & $42.3(29.3-55.3)$ & $22.7(10.6-34.6)$ \\
\hline
\end{tabular}

algae enhancing the vegetal food available to herbivores in seagrass beds (Montgomery and Gerking 1980, Moncreiff and Sullivan 2001).

Some transient fishes had different feeding habits according to the location of the mangrove site (offshore mangrove islet or coastal fringing mangroves). This can be due to daynight migrations during which fish species (such as Lutjanidae) forage in mangroves during the day and in
Este empobrecimiento en ${ }^{13} \mathrm{C}$ también ha sido observado en algunos invertebrados (e.g., anélidos, anfípodos, cangrejos), lo que sugiere que sus fuentes de alimento provienen de los manglares (Christensen et al. 2001, Bouillon et al. 2002, Nagelkerken y van der Velde 2004b). Las especies de la familia Littorinidae se caracterizan por presentar valores bajos de nitrógeno (Christensen et al. 2001, este estudio). Este patrón se podría deber al consumo de epifitas 
seagrass beds at night (Nagelkerken and van der Velde 2004b, Nagelkerken et al. 2006). However, this does not seem to be the case in GCSM as few nocturnal migrations of fishes occur between mangroves and seagrass beds (Kopp et al. 2007), which concurs with the low contribution of mangrove prey to the diet of $L$. apodus (this study). Coastal mangroves can be considered open systems where the availability and exchange of allochthonous sources from seagrass beds, rivers, coral reefs or the lagoon in general can reduce the consumption of autochthonous food resources by transient species (Thimdee et al. 2004, Bouillon et al. 2008, Nyunja et al. 2009). However, it would be interesting to consider other sampling sites in fringing mangroves far from the river mouth to confirm this hypothesis.

Fishes can experience a change in diet during ontogenetic migrations between mangroves and seagrass beds (Nagelkerken and van der Velde 2004b, Lugendo et al. 2006). That was observed in the present study for O. chrysurus, with a slight decrease in mangrove food sources in the diets of large juveniles. Small juveniles of O. chrysurus foraged on zooplankton while larger juveniles consumed larger prey such as crabs and fishes (Cocheret de la Morinière et al. 2003b). This ontogenetic shift in the diet of $O$. chrysurus is confirmed by an increasing gradient in $\delta^{15} \mathrm{~N}$ values, reflecting a variation of the trophic level of this fish species during its life-cycle migration (Cocheret de la Morinière et al. 2003b, present study), as commonly observed in previous studies (Frédérich et al. 2010).

This study revealed that juvenile transient fishes in mangroves mostly foraged in adjacent seagrass beds. Thus, mangroves act more as shelters than feeding grounds. These findings coincide with those of Thimdee et al. (2004), Lugendo et al. (2007) and Vaslet et al. (2012), who showed that mangrove fishes can adopt different feeding behaviors related to mangrove settings (i.e., shoreline mangrove, enclosed mangrove lagoons, and ponds) and prey availability. As mangroves and seagrass beds constitute important ecological fish habitats by acting as either shelters, nurseries or feeding areas, it is of primary importance to preserve these interlinked ecosystems to sustain coastal fisheries.

\section{ACKNOWLEDGMENTS}

We thank the National Park of Guadeloupe for allowing the collection of samples inside the mangrove marine reserve of Fajou islet and Grande Rivière à Goyaves. We are grateful to R Hamparian for his help during the field study. The authors thank the anonymous reviewers for their remarks that improved the manuscript.

\section{REFERENCES}

Aguirre-León A, Yáñez-Arancibia A. 1984. Las mojarras de la laguna de Términos: Taxonomía, biología, ecología y dinámica trófica (Pisces: Gerreidae). An. Inst. Cienc. Mar. Limnol. 9: 150-213. sumergidas muy empobrecidas en ${ }^{15} \mathrm{~N}$ (Christensen et al. 2001, Bouillon et al. 2004). Estas epifitas incorporan fuentes de nitrógeno empobrecidas, tales como $\mathrm{N}_{2}$ o amoníaco volatilizado producido por la remineralización de material del manglar (Bouillon et al. 2004, Fogel et al. 2008).

Se distinguieron dos grupos de peces transeúntes en GCSM según su $\delta^{13} \mathrm{C}$ : la mayoría de los peces presentaron valores de enriquecimiento de carbono similares a las de las fuentes de alimento provenientes de los pastos marinos y sólo unos cuantos especímenes de peces presentaron valores empobrecidos de carbono, lo que sugiere que estos especímenes complementan sus dietas con componentes de los manglares. Los alimentos proporcionados por los manglares fueron consumidos por las especies residentes de los manglares, como B. ronchus, o por especies que toleran entradas de agua dulce y aguas turbias, como C. undecimalis (Louis 1985, Aguirre-León y Díaz-Ruiz 2000). La entrada de nutrientes y la abundancia de infauna pueden explicar la importancia de las presas provenientes de los manglares en la dieta de algunos peces transeúntes (Rodelli et al. 1984, Lugendo et al. 2007, Thollot et al. 1999).

Para la mayoría de los peces juveniles transeúntes, las praderas de pastos marinos son el principal hábitat de alimentación, pero buscan refugio en los manglares. Estos resultados coinciden con los de otros estudios realizados en la región del Caribe los cuales encontraron que juveniles de peces lora (Scaridae) buscan refugio en manglares y se alimentan de algas epifitas que crecen sobre las hojas de pastos marinos (Moncreiff y Sullivan 2001, Cocheret de la Morinière et al. 2003a, Nagelkerken et al. 2006). Este comportamiento se podría atribuir a una mejor disponibilidad de alimento vegetal para los herbívoros en las praderas de pastos marinos debido al alto valor nutricional y la alta productividad de algas epifitas (Montgomery y Gerking 1980, Moncreiff y Sullivan 2001).

Algunos peces transeúntes presentaron diferentes hábitos de alimentación según la ubicación de los manglares (islote o franja costera). Esto se podría deber a las migraciones diurnas y nocturnas de algunos peces (e.g., Lutjanidae), los cuales buscan alimento en los manglares durante el día y en las praderas de pastos marinos durante la noche (Nagelkerken y van der Velde 2004b, Nagelkerken et al. 2006); esto no parece ser el caso en GCSM ya que se realizan pocas migraciones entre los manglares y los pastos marinos (Kopp et al. 2007) y las presas provenientes de los manglares contribuyeron poco a la dieta de L. apodus (este estudio). Los manglares costeros pueden considerarse sistemas abiertos donde la disponibilidad y el intercambio de fuentes alóctonas de las praderas de pastos marinos, los ríos, los arrecifes y la laguna en general pueden reducir el consumo de alimentos autóctonos por las especies transeúntes (Thimdee et al. 2004, Bouillon et al. 2008, Nyunja et al. 2009); sin embargo, sería necesario estudiar otros sitios de los manglares de borde alejados de la desembocadura del río para confirmar esta hipótesis. 
Aguirre-León A, Díaz-Ruiz S. 2000. Population structure, gonadal maturity and feeding habits of Eugerres plumieri (Gerreidae) in the Pom-Atasta fluvial-deltaic system, Mexico. Cienc. Mar. 26: 253-273.

Assor R. 1988. Hydrologie et sédimentologie du Grand Cul-de-Sac Marin (Guadeloupe, F.W.I.). Ann. Soc. Geol. Nord CVII: 221-238

Austin H, Austin S. 1971. The feeding habits of some juvenile marine fishes from the mangroves in western Puerto Rico. Caribb. J. Sci. 11: 171-178.

Beck MW, Heck KLJ, Able KW, Childers DL, Eggleston DB, Gillanders BM, Halpern B, Hays C, Hoshino K, Minello TJ, Orth RJ, Sheridan PF,Weinstein MP. 2001. The identification, conservation and management of estuarine and marine nurseries for fish and invertebrates. BioScience 51: 633-641.

Bosley KL, Wainright SC. 1999. Effects of preservatives and acidification on the stable isotope ratios $\left({ }^{15} \mathrm{~N}:{ }^{14} \mathrm{~N},{ }^{13} \mathrm{C}:{ }^{12} \mathrm{C}\right)$ of two species of marine animals. Can. J. Fish Aquat. Sci. 56: 2181-2185.

Bouchon-Navaro Y, Louis M, Bouchon C. 1992. L'ichtyofaune des herbiers de Phanérogames marines de la baie de Fort-de-France (Martinique, Antilles Françaises). Cybium 16: 307-330.

Bouillon S, Koedam N, Raman AV, Dehairs F. 2002. Primary producers sustaining macro-invertebrate communities in intertidal mangrove forests. Oecologia 130: 441-448.

Bouillon S, Moens T, Overmeer I, Koedam N, Dehairs F. 2004. Resource utilization patterns of epifauna from mangrove forests with contrasting inputs of local versus imported organic matter. Mar. Ecol. Prog. Ser. 278: 77-88.

Bouillon S, Connolly RM, Lee SY. 2008. Organic matter exchange and cycling in mangrove ecosystems: Recent insights from stable isotopes studies. J. Sea Res. 59: 44-58.

Chauvaud S, Bouchon C, Manière R. 2001. Thematic mapping of tropical marine communities (coral reefs, seagrass beds and mangroves) using SPOT data in Guadeloupe Island. Oceanol. Acta 24: 3-16.

Chaves PT, Vendel AL. 2008. Análise comparativa da alimentação de peixes (Teleostei) entre ambientes de marisma e de manguezal num estuário do sul do Brasil (Baía de Guaratuba, Paraná). Rev. Bras. Zool. 25:10-15.

Chiaverini AP. 2008. Ecologia trófica de Sphoeroides testudineus Linnaeus, 1758 e Sphoeroides greeleyi Gilbert, 1900 da gamboa do Perequê, Pontal de Sul, Paraná, Brasil. PhD thesis, Paraná University, Brazil, 54 pp.

Christensen JT, Sauriau PG, Richard P, Jensen PD. 2001. Diet in mangrove snails: Preliminary data on gut contents and stable isotope analysis. J. Shellfish Res. 20: 423-426.

Cocheret de la Morinière E, Pollux BJA, Nagelkerken I, Hemminga MA, Huiskes AH, van der Velde G. 2003a. Ontogenic dietary changes of coral reef fishes in the mangrove-seagrass-reef continuum: Stable isotopes and gut-content analysis. Estuar. Coast. Shelf Sci. 246: 279-289.

Cocheret de la Morinière E, Pollux BJA, Nagelkerken I, van der Velde G. 2003b. Diet shifts of Caribbean grunts (Haemulidae) and snappers (Lutjanidae) and the relation with nursery-to-coral reef migrations. Mar. Ecol. Prog. Ser. 57: 1079-1089.

Ewel KC, Twilley RR, Ong JE. 1998. Different kinds of mangrove forests provide different goods and services. Isot. Environ. Health Stud. 7: 83-94.

Fogel ML, Woller MJ, Cheeseman J, Smallwood BJ, Roberts Q, Meyers MJ. 2008. Unusually negative nitrogen isotopic compositions $\left(\delta^{15} \mathrm{~N}\right)$ of mangroves and lichens in an oligotrophic, microbially-influenced ecosystem. Biogeosciences 5: 1693-1704.
Los peces pueden experimentar un cambio dietético durante las migraciones ontogenéticas entre manglares y praderas de pastos marinos (Nagelkerken y van der Velde 2004b, Lugendo et al. 2006). Esto se observó en el presente trabajo para $O$. chrysurus, registrándose una ligera disminución en las fuentes de alimento provenientes de los manglares en las dietas de los juveniles de mayor tamaño. Los juveniles pequeños de $O$. chrysurus consumieron zooplancton mientras que los de mayor talla consumieron presas más grandes como cangrejos y peces (Cocheret de la Morinière et al. 2003b). El incremento del gradiente de los valores de $\delta^{15} \mathrm{~N}$ confirma este cambio ontogenético en la dieta de O. chrysurus, y refleja una variación del nivel trófico de esta especie durante la migración en su ciclo de vida (Cocheret de la Morinière et al. 2003b, este estudio), como se ha observado en otros trabajos (Frédérich et al. 2010).

En este estudio se encontró que los peces juveniles transeúntes en los manglares se alimentan en los pastos marinos adyacentes. Esto indica que los manglares actúan más como zona de refugio que como zona de alimentación para los peces. Estos resultados coinciden con los de Thimdee et al. (2004), Lugendo et al. (2007) y Vaslet et al. (2012), quienes mostraron que los peces de manglares pueden adoptar diferentes hábitos de alimentación según la ubicación de los manglares (e.g., bordes costeros, lagunas encerradas) y la disponibilidad de presas. Dado que los manglares y las praderas de pastos marinos son importantes hábitats ecológicos para los peces ya que funcionan como zonas de refugio, crianza y alimentación, es de suma importancia preservar estos ecosistemas interrelacionados para sostener las pesquerías costeras.

\section{AgRadecimientos}

Agradecemos al Parque Nacional de Guadalupe (Antillas Menores) el permiso otorgado para recolectar muestras dentro de la reserva marina de manglares (islote de Fajou y Grande Rivière à Goyaves), a R Hamparian su apoyo durante el trabajo de campo y a los revisores anónimos cuyos comentarios ayudaron a mejorar el manuscrito.

Traducido al español por Christine Harris.

Frédérich B, Lehanse O, Vandewalle P, Lepoint G. 2010. Trophic niche width, shift, and specialization of Dascyllus aruanus in Toliara Lagoon, Madagascar. Copeia 2: 218-226.

Gautier F. 2015. Function of Thalassia testudinum seagrass food webs in Guadeloupe (Lesser Antilles): an approach with stable isotopes and fatty acid biomarkers. $\mathrm{PhD}$ thesis, University of the Lesser Antilles, Guadeloupe, 208 pp.

Gearing JN. 1991. The study of diet and trophic relationships through natural abundance ${ }^{13} \mathrm{C}$. In: Coleman DC, Fry B (eds.), Carbon Isotope Techniques. Academic Press, pp. 201-218.

Gilmore RG, Snedaker SC. 1993. Mangrove forests. In: Martin WH, Boyce SG, Echternacht AC (eds.), Biodiversity of the 
Southeastern United States, Lowland Terrestrial Communities. John Wiley, pp. 165-198.

Granek EF, Compton JE, Phillips DL. 2009. Mangrove-exported nutrient incorporation by sessile coral reef invertebrates. Ecosystems 12: 462-472.

Heithaus ER, Heithaus PA, Heithaus MR, Burkholder D, Layman CA. 2011. Trophic dynamics in a relative pristine subtropical fringing mangrove community. Mar. Ecol. Prog. Ser. 428: 49-61.

Hemminga MA, Mateo MA. 1996. Stable carbon isotopes in seagrasses: Variability in ratios and use in ecological studies. Mar. Ecol. Prog. Ser. 140: 285-298.

Kieckbusch DK, Koch MS, Serafy JE, Anderson WT. 2004. Trophic linkages among primary producers and consumers in fringing mangroves of subtropical lagoons. Bull. Mar. Sci. 74: 271-285.

Kopp D, Bouchon-Navaro Y, Louis M, Bouchon C. 2007. Diel differences in the seagrass fish assemblages of a Caribbean island in relation to adjacent habitat types. Aquat. Bot. 87: 31-37.

Laegdsgaard P, Johnson C. 2001. Why do juvenile fish utilise mangrove habitats? J. Exp. Mar. Biol. Ecol. 257: 229-253.

Legendre L, Legendre P. 1998. Numerical Ecology. Elsevier Science, Amsterdam, 853 pp.

Louis M. 1985. Reproduction and growth of Bairdiella ronchus (Fish Sciaenidae) in the mangroves of Guadeloupe (Lesser Antilles). Rev. Hydrobiol. Trop. 18: 61-72.

Lugendo BR, Nagelkerken I, van der Velde G, Mgaya YD. 2006. The importance of mangroves, mud and sand flats, and seagrass beds as feeding areas for juvenile fishes in Chwaka Bay, Zanzibar: Gut content and stable isotope analyses. J. Fish Biol. 69: 1639-1661.

Lugendo BR, Nagelkerken I, Kruitwagen G, van der Velde G, Mgaya YD. 2007. Relative importance of mangroves as feeding habitats for fishes: A comparison between mangrove habitats with different settings. Bull. Mar. Sci. 80: 497-512.

Marguillier S, van der Velde G, Dehairs F, Hemminga MA, Rajagopal S. 1997. Trophic relationships in an interlinked mangrove-seagrass ecosystem as traced by $\delta^{13} \mathrm{C}$ and $\delta^{15} \mathrm{~N}$. Mar. Ecol. Prog. Ser. 151: 115-121.

McCutchan JH, Lewis WM, Kendall C, McGrath CC. 2003. Variation in trophic shift for stable isotope ratios of carbon, nitrogen, and sulfur. Oikos 102: 378-390.

Moncreiff CA, Sullivan MJ. 2001. Trophic importance of epiphytic algae in subtropical seagrass beds: Evidence from multiple stable isotope analyses. Mar. Ecol. Prog. Ser. 215: 93-106.

Montgomery WL, Gerking SD. 1980. Marine macroalgae as foods for fishes: An evaluation of potential food quality. Environ. Biol. Fish 5: 143-153.

Nagelkerken I, van der Velde G. 2004a. Are Caribbean mangroves important feeding grounds for juvenile reef fish from adjacent seagrass beds? Mar. Ecol. Prog. Ser. 274: 143-151.

Nagelkerken I, van der Velde G. 2004b. Relative importance of interlinked mangroves and seagrass beds as feeding habitats for juvenile reef fish on a Caribbean island. Mar. Ecol. Prog. Ser. 274: $153-159$.

Nagelkerken I, Dorenbosch M, Verberk WCEP, Cocheret de la Morinière E, van der Velde G. 2000. Day-night shifts of fishes between shallow-water biotopes of a Caribbean bay, with emphasis on the nocturnal feeding of Haemulidae and Lutjanidae. Mar. Ecol. Prog. Ser. 194: 55-64.

Nagelkerken I., van der Velde G., Verberk WCEP, Dorenbosch M. 2006. Segregation along multiple resource axes in a tropical seagrass fish community. Mar. Ecol. Prog. Ser. 308: 79-89.

Nagelkerken I, Blaber SJM, Bouillon S, Green P, Haywood M, Kirton LG, Meynecke JO, Pawlik J, Penrose HM, Sasekumar A,
Somerfield PJ. 2008. The habitat function of mangroves for terrestrial and marine fauna: A review. Aquat. Bot. 89: 155-185.

Nyunja J, Ntiba M, Onyari J, Mavuti K, Soetaert K, Bouillon S. 2009. Carbon sources supporting a diverse fish community in a tropical coastal ecosystem (Gazi Bay, Kenya). Estuar. Coast. Shelf Sci. 83: 333-341.

Parnell AC, Inger R, Bearhop S, Jackson AL. 2010. Source partitioning using stable isotopes: Coping with too much variation. PlosOne 5: 1-5.

Peterson BJ, Fry B. 1987. Stable isotopes in ecosystem studies. Annu. Rev. Ecol. Syst. 18: 293-320.

Post DM, Layman CA, Arrington DA, Takimoto G. 2007. Getting to the fat of the matter: Models, methods and assumptions for dealing with lipids in stable isotope analyses. Oecologia 152: 179-189.

R Development Core Team. 2012. R: A Language and Environment for Statistical Computing. Version 2.15.0 ${ }^{\circledR}$.

Randall JE. 1967. Food habits of reef fishes of the West Indies. Stud. Trop. Oceanogr. Miami 5: 665-847.

Raven JA, Johnston AM, Kübler JE, Korb R, McInroy SG, Handley LL, Scrimgeour CM, Walker DI, Beardall J, Vanderklift M, Fredriksen S, Dunton KH. 2002. Mechanistic interpretation of carbon isotope discrimination by marine macroalgae and seagrasses. Funct. Plant Biol. 29: 355-378.

Rodelli MR, Gearing JN, Marshall N, Sasekumar A. 1984. Stable isotope ratio as a tracer of mangrove carbon in Malaysian ecosystems. Oecologia 61: 326-333.

Sheaves M, Molony B. 2000. Short-circuit in the mangrove food chain. Mar. Ecol. Prog. Ser. 199: 97-109.

Sierra LM, Claro R, Popovo OA. 1994. Alimentación y relaciones tróficas. In: Claro R (ed.), Ecología de los Peces Marinas de Cuba. Academia de Ciencas de Cuba y Centro de Investigaciones de Quintana Roo, pp. 263-319.

Sweeting CJ, Polunin NVC, Jennings S. 2006. Effects of chemical lipid extraction and arithmetic lipid correction on stable isotope ratios of fish tissues. Rapid Commun. Mass Spectrom. 20: 595-601.

Thimdee W, Deein G, Sangrungruang C, Matsunaga K. 2004. Analysis of primary food sources and trophic relationships of aquatic animals in a mangrove-fringed estuary, Khung Krabaen Bay (Thailand), using dual stable isotope techniques. Wetlands Ecol. Manage. 12: 135-144.

Thollot P, Kulbicki M, Harmelin-Vivien M. 1999. Trophic analysis and food webs of mangrove fish assemblages from New Caledonia. C.R. Acad. Sci. Paris, Sci. Vie 322: 607-619.

Vander Zanden MJ, Rasmussen JB. 2001. Variation in $\delta^{15} \mathrm{~N}$ and $\delta^{13} \mathrm{C}$ trophic fractionation: Implications for aquatic food web studies. Limnol. Oceanogr. 46: 2061-2066.

Vaslet A. 2009. Mangrove fish communities in the lesser Antilles: influence of environmental variables and isotopic approach of food webs. $\mathrm{PhD}$ thesis, University of the Lesser Antilles, Guadeloupe, $274 \mathrm{pp}$.

Vaslet A, Bouchon-Navaro Y, Charrier G, Louis M, Bouchon C. 2010. Spatial patterns of mangrove shoreline fish communities in relation with environmental variables in Caribbean lagoons. Estuar. Coasts 33: 195-210.

Vaslet A, Phillips DL, France C, Feller IC, Baldwin CC. 2012. The relative importance of mangroves and seagrass beds as feeding areas for resident and transient fishes among different mangrove habitats in Florida and Belize: Evidence from dietary and stableisotope analyses. J. Exp. Mar. Biol. Ecol. 434-435: 81-93.

Received November 2014, accepted July 2015. 